\title{
Statistical Analysis of the Acceleration Zone Location in Hall Thrusters
}

\author{
Jesse A. Linnell ${ }^{1}$ and Alec D. Gallimore ${ }^{2}$ \\ University of Michigan, Ann Arbor, MI 48109 USA
}

\begin{abstract}
This study uses a stepwise regression strategy to examine the interaction between the Hall thruster operating parameters and the location of the acceleration zone. The results of the regression are then linked to the Hall thruster physics, specifically the cross-field electron mobility. The location of the acceleration zone is shown to have a strong correlation with peak magnetic field strength and a correlation with a two-factor interaction between discharge current and peak magnetic field strength. These terms are related to Bohm and classical electron mobility, respectively. As peak magnetic field strength increase, the acceleration zone moves upstream. As discharge current increase the acceleration zone moves downstream. The acceleration zone starts where the centerline magnetic field is between $70-90 \%$ of the peak centerline magnetic field strength. This has implications for estimating the acceleration zone location, the relative beam divergence, and the location of discharge channel erosion during thruster design.
\end{abstract}

\section{Introduction}

A LTHOUGH a fundamental aspect of Hall thruster physics, the establishment of the acceleration zone is a poorly understood phenomenon. In Hall thrusters, the acceleration zone is the region in which ionized propellant is accelerated and thrust is produced. The location and shape of the acceleration zone is an important design consideration because it can strongly affect the beam divergence of the thruster plume. This has important implications for the thrust efficiency and plume-spacecraft interactions. The location of the acceleration zone also plays a role in Hall thruster lifetimes by dictating the location of discharge channel erosion. Furthermore, improved beam focusing is an important design consideration for krypton propellant, which has been observed to suffers from poor beam divergence. ${ }^{1}$

Internal measurements of the NASA-173Mv1 Hall thruster with a floating emissive probe have been used to characterize the acceleration zone., ${ }^{2,3}$ Theses studies show that krypton's poor beam divergence is attributed to the shape and location of the acceleration zone. Also observed in reference 2 is that at higher discharge voltage the acceleration zone begins farther upstream and has more focused equipotential lines. However, the precise cause of these trends is not fully explained. It is the intention of this analysis to take the first steps in addressing these unanswered questions. This paper uses a statistical approach and the results from of an internal characterization of the NASA-173Mv1 Hall thruster ${ }^{2,3}$ to study trends in the location of the acceleration zone.

This analysis uses a stepwise regression as an automated tool to find trends between experimental data and operational parameters of the Hall thruster. This analysis builds on the idea that Hall thruster operational parameters directly drive the physics inside the discharge channel. For example, anode flow rate drives the particle number density and discharge voltage drives the electron temperature. Once the regression analysis is complete, the trends are substantiated by relating them back to the Hall thruster physics. This process has the advantage of removing the analyst's biases and presumptions. Another advantage is that the analysis does not rely on the inadequate electron mobility model. Lastly, by dealing with operational parameters of the Hall thruster, the results have a system level perspective, which will aid Hall thruster designers.

\footnotetext{
${ }^{1}$ Technical Staff, MIT Lincoln Laboratory, 244 Wood St, S2-645, Lexington, MA 02420 USA, jlinnell@ll.mit.edu, AIAA Member.

${ }^{2}$ Associate Dean for Academic Programs and Initiatives at the Horace H. Rackham School of Graduate Studies and Director of the Plasmadynamics and Electric Propulsion Laboratory with the Department of Aerospace Engineering, University of Michigan, alec.gallimore@umich.edu, 1919 Green Rd, Room B107, Ann Arbor, MI 48109 USA, Associate Fellow AIAA.
} 
The goal of this analysis is not to propose a new model for electron mobility physics nor is it meant to develop a statistical method. The goals of this paper are to present a novel and simple approach to a difficult engineering problem and to find general rules of thumb that can be used by Hall thruster designers. This analysis identifies large scale trends in these data and is meant to represent the first step in a larger analysis effort.

\section{A. Hall Thruster Physics}

Experimental data are taken with the NASA-173Mv1, which is discussed in detail in other papers and will not be covered here. ${ }^{4,5}$ A notional cross-sectional cut of a Hall thruster discharge channel is shown in Fig. 1. There are perpendicular electric and magnetic fields resulting in an azimuthal electron current. The magnetic field is weak near the anode then increases and peaks near the exit of the discharge channel. The plasma potential is fairly flat in the upstream region of the discharge channel until it reaches the region of peak magnetic field strength at which point the plasma potential drops rapidly. This region has a strong axial electric field and is known as the acceleration zone.

As shown by Fig. 1, the magnetic field lines have a concave shape and are approximately symmetric about the centerline of the channel. The magnetic field curvature is higher in the upstream portion of the discharge channel. This field line curvature is referred to as a plasma lens and is shown to be beneficial to Hall thruster performance. ${ }^{1,4,5}$ Beam focusing is improved because to the first order, the equipotential lines follow the magnetic field lines. This has long been suggested ${ }^{6,7}$ and has been experimentally verified in Hall thrusters. ${ }^{2,3}$ This finding is important to Hall thruster designers, but the precise location of the acceleration zone is still unresolved. This study focuses on the location of the acceleration zone and sheds light on this missing piece of information.

To study the acceleration zone one must focus on the electron current density perpendicular to the magnetic field lines. The 1-D Ohm's Law is shown in Eq. (1). In Eq. (1), $j_{e}$ is the axial electron current, $n_{e}$ is the electron number density, $k_{B}$ is the Boltzmann constant, $T_{e}$ is the electron temperature, $e$ is the elementary electron charge, and $\mu$ is the cross-field electron mobility. The cross-field mobility is the main factor determining the electric field. For this reason, operational parameters that affect the cross-field electron mobility are expected to have the largest effect on the location of the acceleration zone.

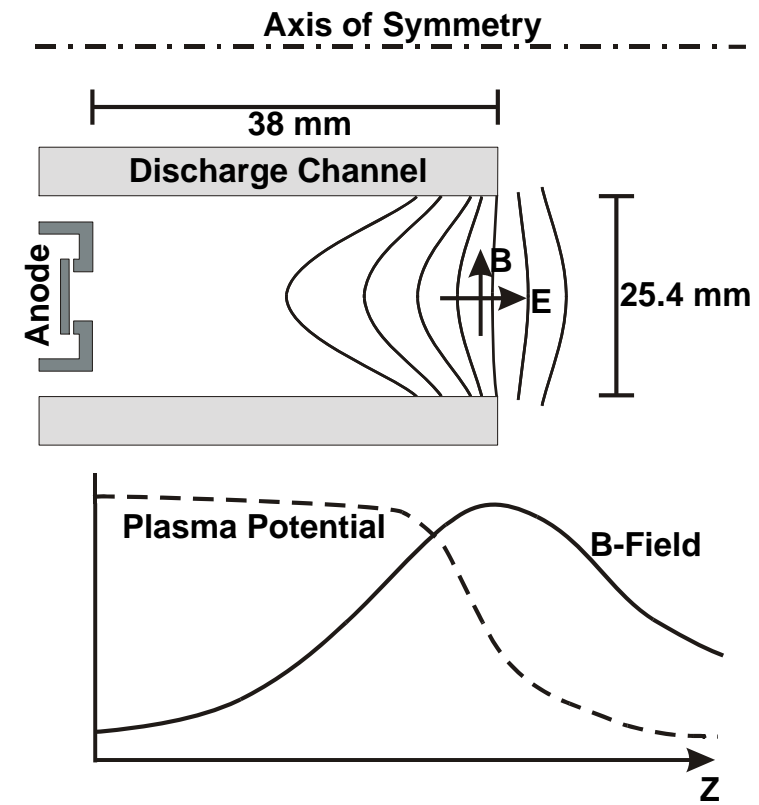

Figure 1. Notional Hall Thruster Discharge Channel

$$
j_{e \perp}=\mu\left(e n_{e} E_{\perp}+k_{B} \nabla_{\perp} n_{e} T_{e}\right)
$$

Traditionally, the cross-field mobility is modeled as a combination of three components: Bohm, classical, and near-wall conductivity. Bohm type mobility, shown in Eq. (2), stems from the turbulent fluctuations in the electric field and plasma density. The variable $\alpha$ is a constant and typically ranges from $10^{-2}-1 / 16 .^{8-10}$ Classical mobility, shown in Eq. (3), occurs when an electron experiences a momentum transfer collision with a heavy particle and undergoes a random-walk process across the magnetic field lines. In Eq. (3), $m_{e}$ is electron mass, $B$ is the magnetic field strength, and $v_{m}$ is the electron-heavy particle collision rate and is defined in Eq. (4). In Eq. (4), $V_{e}$ is the electron velocity, $n_{i}$ is the ion number density, $n_{n}$ is the neutral number density, $Q_{e i}$ is the electron-ion collision cross section, and $Q_{e n}$ is the electron-neutral collision cross section. Near-wall conductivity proposes that electron collisions with the walls enhance electron cross-field mobility and in this way the walls act as a macro-particle. This type of mobility cannot be addressed in this analysis and will not be the focus of discussion. 


$$
\begin{gathered}
\mu_{B}=\frac{1}{\alpha B} \\
\mu_{c} \approx \frac{m_{e} v_{m}}{e B^{2}} \\
v_{m}=n_{i}\left\langle Q_{e i} V_{e}\right\rangle+n_{n}\left\langle Q_{e n} V_{e}\right\rangle
\end{gathered}
$$

Several of the variables shown in Eq. (1) can be related back to the operating conditions of the thruster. Discharge voltage acts to increase electron temperature and the strength of the electric field. Anode flow rate acts to increase the plasma density and the electron-heavy particle collision frequency. The strength of the magnetic field is of particular importance because it drives both the Bohm and classical mobility models. Other factors such as discharge current oscillations, plasma-wall interactions, propellant type, discharge channel dimensions, and magnetic field shape also have important effects on the axial electron current.

Although these electron transport phenomena are accepted by the modeling community, the existing transport models are flawed and only produce reasonable results with liberal manipulation from the modeler. For this reason, this analysis will try to separate itself from the flawed electron transport model and approach the problem from a different angle.

\section{B. Regression Analysis}

This analysis uses a stepwise regression ${ }^{11,12}$ and is conducted using the stepwise regression function in the MATLAB statistics toolbox. Stepwise regression is a multiple regression technique where input variables are iteratively added or removed based on their statistical significance. Statistical significance is determined by the Pvalue, which is calculated for all variables in each iterative step. If the P-value meets a critical value, the variable is added or removed from the model. The critical value for addition is P-value $<0.03$ and the critical value for removal is P-value $>0.1$. Stepping is terminated when no variables meet the entry or removal criteria. The error in the response variable is assumed to be homoscedastic. For this analysis, the input variables are the operational parameters of the Hall thruster and a simple characterization of the magnetic field. The response variables are the experimentally measured features of the acceleration zone.

This type of analysis is often considered controversial because it removes the human element from the regression analysis. For this reason, the results must be validated by relating them to accepted Hall thruster physics (Eqs. (1)(4)). A common complication with this type of analysis is the effect of collinearity in the data. Care must be taken when more than one input variable tends to vary together. For example, in order to optimize thruster efficiency magnetic field strength must increase with discharge voltage and anode flow rate.

This analysis includes both one-factor and two-factor interactions as input parameters. One-factor effects are those driven by a single variable (e.g. A, B, C). Two-factor interactions are used to describe the interaction between two of the one-factor variables (e.g. AB, BC, AC). Also, the text refers to a "primary factor", which is the variable that has the most statistically significant relationship with the response variable understudy. In this analysis, all one and two-factor interactions are considered and no effort is made to accept or reject the variables prior to analysis.

For this regression analysis the Hall thruster is viewed from a systems perspective and can be seen in Fig. 2. The thruster input factors, control factors, and noise factors drive the physics inside the Hall thruster. In response, the Hall thruster performance varies. The input factors are variables that are modified during the experiment. For this analysis the input factors are the thruster operation parameters. Control factors are variables that remain fixed and in this case correspond to design parameters such as dimensions and materials. Noise factors are variables out of analyst's control such as environmental and manufacturing variations.

For this analysis, operation parameters are varied and a total of 21 variables are considered. There are six onefactor interactions including: propellant type, discharge voltage $\left(V_{d}\right)$, discharge current $\left(I_{d}\right)$, trim coil current $\left(I_{T C}\right)$, the normalized peak magnetic field strength $\left(B_{\max }\right)$, and mass flow rate. There are also 15 two-factor interactions. Some examples of these are: $V_{d} I_{d}, I_{d} I_{T C}, I_{d} \cdot B_{\max }$, etc. Variables such as the cathode potential and anode efficiency are reported, but are not used in the analysis because they are considered response variables.

Discharge current is dictated predominantly by the anode flow rate and to second order by discharge voltage and the magnet coil settings. In general, discharge current can be considered a response variable. However, as shown in Eq. (1), number density is an important value of interest. Because of krypton's smaller atomic mass it has a $25 \%$ higher velocity and accordingly a 25\% lower discharge channel number density than xenon at matched volumetric flow rate. When xenon and krypton operate at the same discharge current, krypton requires approximately 25\% 
larger volumetric flow rate than xenon and the heavy particle number density is conveniently well matched. For this reason, there is a particular advantage when comparing xenon and krypton operation at matched discharge current. If only xenon data points are taken, the anode flow rate would be sufficient for the regression analysis. Both values are included for completeness.

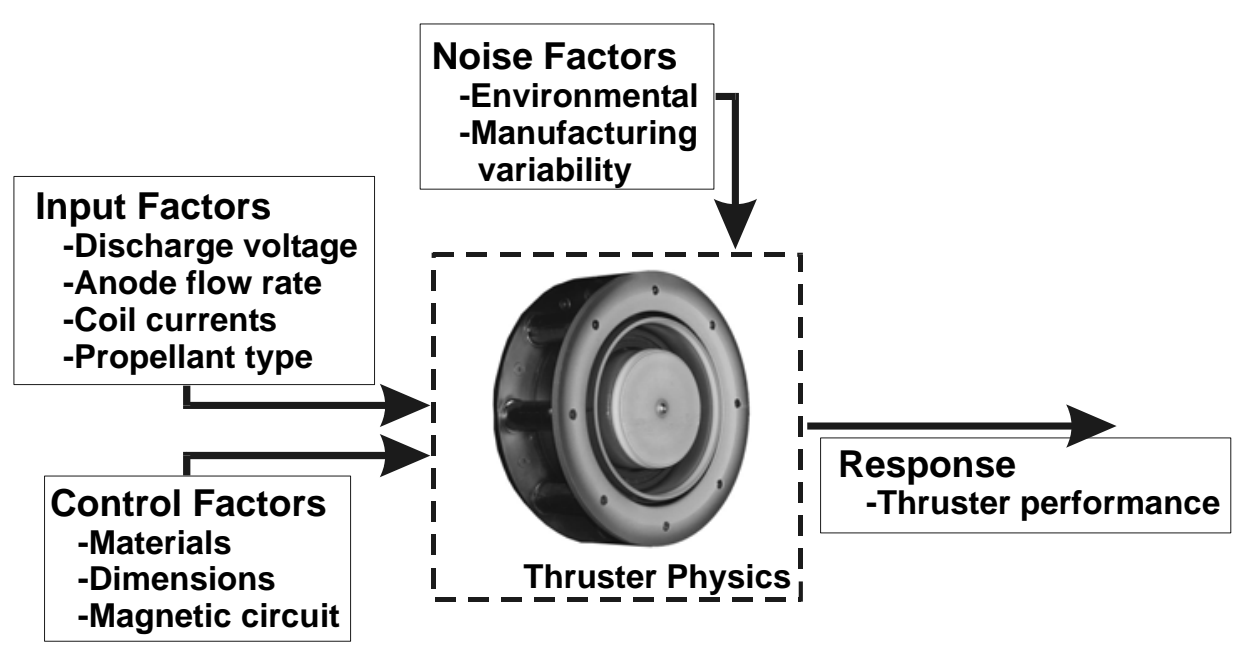

Figure 2. Parameter Diagram of a Hall Thruster

The magnetic field is the most difficult parameter to characterize in simple terms. For this analysis, the magnetic field has been characterized by the peak centerline magnetic field and the trim coil current. The peak centerline magnetic field is dictated by the inner and outer coil currents and the trim coil current is used as a rough measure of the magnetic field curvature. As the trim coil current increases, so does the axial gradient in radial magnetic field and the magnetic field curvature.

These values are sufficient for characterizing the magnetic field since the same Hall thruster is used for all measurements and the magnetic field structure near the thruster exit is similar in all cases. For example, the magnetic field topology is symmetric in all cases and the location of the peak magnetic field is fixed. Near the discharge channel exit the magnetic field is shaped by the magnetic circuit, specifically the magnetic screens, the inner coil, and the outer coil. The trim coil drives the shape of the magnetic field near the anode.

Hall thruster dimensions ${ }^{13}$ and materials may also have an effect on the acceleration zone location in Hall thrusters, but these trends cannot be addressed with the current analysis because only one thruster is studied. Although, the exact results of this analysis will not be identical for all thrusters, the large scale trends will be consistent.

\section{Acceleration Zone Trends}

The operating conditions and the internal characteristics for the NASA-173Mv1 can be seen in Table I. Two types of propellant were used in this study and are represented in the regression analysis by the values of 1 and -1 for xenon and krypton, respectively. The acceleration zone start location on the centerline is measured using a floating emissive probe. ${ }^{2,3}$ The acceleration zone is defined as the region where the axial electric field is greater than $15 \%$ of the peak axial electric field. The $15 \%$ threshold is used to avoid false detections of the acceleration zone boundaries because it is slightly larger than the noise in these axial electric field data. The magnetic field is modeled using the 3D magnetostatic solver Magnet 6.0 by Infolytica. The peak centerline magnetic field is normalized and reported in Table I. In accordance with International Traffic and Arms Regulations (ITAR) the magnetic field has also been normalized by an arbitrarily chosen value of $\gamma$. The magnetic field at the start of the acceleration zone is divided by the peak centerline magnetic field and is reported in Table I under the heading "B-Fraction at Start".

Due to the difficult nature of internal Hall thruster characterization the number of data points are limited to 14 . Ideally, experimental data would be systematically varied over all operation parameters and taken for a number of different thrusters. Because of the modest data set, the regression analysis is somewhat limited and the coefficients calculated in the regression equations will be inexact. However, it is possible to identify the dominant variables that 
define the location of the acceleration zone. This analysis represents the first step in an analysis effort and should successfully identify the large scale trends for design of future experiments.

Table I. Data Points from the Internal Hall Thruster Investigation

\begin{tabular}{|c|c|c|c|c|c|c|c|c|c|c|c|}
\hline Propellant & $\begin{array}{c}\text { Cathode } \\
\text { Potential, } \\
\text { V }\end{array}$ & $\begin{array}{l}V_{d}, \\
\mathrm{~V}\end{array}$ & $\begin{array}{c}I_{d} \\
\mathrm{~A}\end{array}$ & $\begin{array}{c}\text { Anode } \\
\text { Flow, } \\
\mathrm{mg} / \mathrm{s}\end{array}$ & $\begin{array}{c}\text { Anode } \\
\text { Effic., } \\
\%\end{array}$ & $\begin{array}{l}\text { Inner } \\
\text { Coil, } \\
\text { A }\end{array}$ & $\begin{array}{c}\text { Outer } \\
\text { Coil, } \\
\text { A }\end{array}$ & $\begin{array}{c}\text { Trim } \\
\text { Coil, } \\
\text { A }\end{array}$ & $\begin{array}{l}\text { Normalized } \\
\text { Peak B-field }\end{array}$ & $\begin{array}{c}\text { Accel. } \\
\text { Zone } \\
\text { Start, mm }\end{array}$ & $\begin{array}{c}\text { B-Fraction } \\
\text { at Start }\end{array}$ \\
\hline $\mathrm{Xe}$ & -10.5 & 300 & 9.17 & 10 & 57.9 & 1.89 & 2.21 & 0 & 1.11 & 35.2 & 0.91 \\
\hline $\mathrm{Xe}$ & -11.5 & 500 & 9.35 & 10 & 61.6 & 2.9 & 2.67 & 0 & 1.52 & 30.8 & 0.74 \\
\hline $\mathrm{Xe}$ & -11.4 & 600 & 9.55 & 10 & 60.9 & 2.73 & 2.72 & 0 & 1.48 & 32.7 & 0.82 \\
\hline $\mathrm{Xe}$ & -10.9 & 300 & 8.95 & 10 & 61.2 & 1.88 & 2.21 & -0.51 & 1.04 & 34.15 & 0.85 \\
\hline $\mathrm{Xe}$ & -11.7 & 500 & 9.27 & 10 & 66.1 & 2.9 & 2.87 & -0.87 & 1.45 & 31.4 & 0.72 \\
\hline $\mathrm{Xe}$ & -12.3 & 600 & 9.59 & 10 & 64.8 & 3.17 & 3.42 & -1.08 & 1.64 & 32 & 0.74 \\
\hline $\mathrm{Kr}$ & -14.8 & 500 & 7.78 & 6.38 & 47.7 & 1.57 & 2.16 & 0 & 1.01 & 33.7 & 0.86 \\
\hline $\mathrm{Kr}$ & - & 500 & 9.39 & 7.35 & 49.9 & 1.79 & 2.18 & 0 & 1.08 & 35.05 & 0.90 \\
\hline $\mathrm{Kr}$ & -14.8 & 600 & 7.84 & 6.38 & 53.5 & 1.65 & 2.26 & 0 & 1.08 & 32.8 & 0.83 \\
\hline $\mathrm{Kr}$ & -13.3 & 600 & 9.55 & 7.36 & 51.4 & 1.98 & 2.18 & 0 & 1.13 & 34.65 & 0.89 \\
\hline $\mathrm{Kr}$ & -14.2 & 500 & 7.54 & 6.38 & 51.6 & 1.57 & 2.17 & -0.41 & 0.95 & 34.55 & 0.87 \\
\hline $\mathrm{Kr}$ & -14.4 & 500 & 9.27 & 7.77 & 57.2 & 1.79 & 2.27 & -0.43 & 1.04 & 34.8 & 0.88 \\
\hline $\mathrm{Kr}$ & -14.6 & 600 & 7.49 & 6.38 & 57.9 & 1.65 & 2.26 & -0.19 & 1.03 & 34.3 & 0.87 \\
\hline $\mathrm{Kr}$ & -13.3 & 600 & 9.59 & 7.8 & 56.1 & 1.98 & 2.18 & -0.46 & 1.07 & 37.15 & 0.91 \\
\hline
\end{tabular}

\section{A. Acceleration Zone Location Regression Analysis}

The response variable in this section is acceleration zone start location. The equations resulting from the stepwise regression appear below. The regression equation using only the primary variable (normalized peak magnetic field) is given in Eq. (5), the regression equation including all statistically significant one-factor interactions is given in Eq. (6), and the regression equation including all statistically significant one and two-factor interaction is given in Eq. (7). In these equations, $Z_{a c c e l}$ is the location of the acceleration zone start and $a-c$ are coefficients determined by the multiple regression analysis. The $a, b$, and $c$ coefficients are given with the corresponding 95\% confidence intervals (CI). Statistical results for the stepwise regression are shown in Table II. The experimental results and regression equations can be seen in Fig. 3 plotted against the primary variable.

$$
\begin{aligned}
& Z_{\text {Accel }}=a \frac{B_{\max }}{\gamma}+c \\
& a=-5.51, \quad 95 \% C I:-8.62 \text { to }-2.41 \\
& c=40.34, \quad 95 \% C I: 36.60 \text { to } 44.09 \\
& Z_{\text {Accel }}=a \frac{B_{\max }}{\gamma}+b I_{d}+c \\
& a=-7.88, \quad 95 \% C I:-10.67 \text { to }-5.09 \\
& b=1.18, \quad 95 \% C I: 0.40 \text { to } 1.96 \\
& c=32.68, \quad 95 \% C I: 26.89 \text { to } 38.47 \\
& Z_{\text {Accel }}=a \frac{B_{\max }}{\gamma}+b \frac{B_{\max }}{\gamma} I_{d}+c \\
& a=-19.41, \quad 95 \% C I:-28.19 \text { to }-10.63 \\
& b=1.21, \quad 95 \% C I: 0.47 \text { to } 1.95 \\
& c=43.97, \quad 95 \% C I: 40.50 \text { to } 47.44
\end{aligned}
$$


As shown in Eq. (5), the variable with the most statistically significant variation with acceleration zone location is the peak magnetic field strength and accounts for $56 \%$ of the variation in these data. This result is intuitive because both Bohm and classical electron mobility (refer to Eq. (2) and Eq. (3)) are functions of magnetic field strength. As magnetic field strength increases, the cross-field electron mobility decreases and the acceleration zone moves upstream. This trend is also observed by Yamamoto. $^{14}$

If all one-factor interactions are included, the regression analysis also identifies discharge current as a statistically significant variable (refer to Eq. (6)). As discharge current increases, the heavy particle number density increases, resulting in higher electron mobility and the acceleration zone moves downstream. This downstream shift of acceleration zone with increased discharge current has been both modeled ${ }^{15}$ and measured experimentally. ${ }^{16,17}$ With all one-factor interactions included in the regression, the effect of classical and Bohm electron mobility begins to distinguish themselves from one another.

As all one and two-factor interactions are included in the regression analysis, the effect of Bohm mobility can still be seen in the first term but the form of classical mobility term is altered (refer to Eq. (7)). Discharge current alone is no longer identified as a statistically significant variable and is replaced by the two-factor interaction between discharge current and peak magnetic field strength. The term shows a coupling between discharge current and magnetic field strength and more closely resembles the relation given in Eq. (3). As the two-factor term increases, the acceleration zone moves downstream. The one and two-factor interaction equation accounts for $80 \%$ of the variation observed in these data.

Although this analysis can identify coupling between discharge current and magnetic field strength, it does not differentiate between magnetic field raised to the power of 1 or -1 (i.e. $I_{d} B$ and $I_{d} / B$ ). This is where physical interpretation of the results is important. Also, this data set cannot distinguish between the effects of $B^{-1}$ and $B^{-2}$. Most of these data shown in Table I fall in two general areas around $B_{\max }=1.05$ and 1.5. Just as one cannot define a parabola with two points, it is not possible to differentiate magnetic field scaling of 1 or 2 with these data. Given data taken oven incremental steps of magnetic field strength this differentiation would be possible.

These trends can help to inform designers about the major factors that drive the location of the acceleration zone. This also helps to describe trends observed in references. ${ }^{2,3}$ The most significant variables driving the location of the acceleration zone is strength of the magnetic field and discharge current. As mentioned before, these variables account for $80 \%$ of the variation in these data. Propellant type and discharge voltage are found not to have a statistically significant effect on acceleration zone location. Although the number of data points in Table I is small, the discharge voltage is well varied in these data so any significant relation between acceleration zone location and discharge voltage would be captured.

Since this analysis is conducted on only one thruster and the data set is not all encompassing, the specific values of the coefficients are not particularly meaningful. The large scale trends are the most meaningful piece of insight taken from this analysis. The next analysis will attempt to find a more meaningful design variable.

\section{B. Magnetic Field Fraction Regression Analysis}

In an attempt to determine a simple design criterion, the fraction of the magnetic field at the start of the acceleration zone is treated as the response variable in the regression analysis. Gavryushin et al. ${ }^{18}$ suggest that the acceleration zone begins approximately where the centerline magnetic field is equal to $80 \%$ of the peak centerline magnetic field. The next regression analysis will test this claim. 
Results from the multiple regression analysis for the primary variable, all one-factor interactions, and all one and two-factor interactions are shown in Eqs. (8), (9), and (10), respectively. In these equations, $X_{B \text {-frac }}$ is the magnetic field fraction, and variables $d-f$ and coefficients from the multiple regressions. The statistical results of the stepwise regression are shown in Table III. The experimental results and the regression equations are shown in Fig. 4 plotted against the primary variable.

$$
\begin{aligned}
& X_{B-\text { frac }}=d \frac{B_{\max }}{\gamma}+f \\
& d=-0.234, \quad 95 \% C I:-0.331 \text { to }-0.137 \\
& f=1.12, \quad 95 \% C I: 1.00 \text { to } 1.24 \\
& X_{B-\text { frac }}=d \frac{B_{\max }}{\gamma}+e I_{d}+f \\
& d=-0.297, \quad 95 \% C I:-0.396 \text { to }-0.198 \\
& e=0.031, \quad 95 \% C I: 0.003 \text { to } 0.059 \\
& f=0.918, \quad 95 \% C I: 0.713 \text { to } 1.124 \\
& X_{B-\text { frac }}=d \frac{B_{\max }}{\gamma}+e \frac{B_{\max }}{\gamma} I_{d}+f \\
& d=-0.603, \quad 95 \% C I:-0.919 \text { to }-0.287 \\
& e=0.032, \quad 95 \% C I: 0.006 \text { to } 0.059 \\
& f=1.22, \quad 95 \% C I: 1.09 \text { to } 1.34
\end{aligned}
$$

For this regression analysis, the peak magnetic field strength accounts for $70 \%$ of the variation in these data and when the two-factor interaction between discharge current and peak magnetic field are included, $82 \%$ of the variation is accounted for. The same Bohm mobility and classical mobility terms are identified by this regression analysis. This is not surprising since there is a strong link between magnetic field fraction and the acceleration zone start location. As stated previously, the location of peak magnetic field is fixed and the basic shape near the exit of the discharge channel is similar for all cases.

For cases with weaker magnetic fields, the acceleration zone is expected to begin at the centerline location with a magnetic field fraction of $90 \%$. For cases with larger magnetic field, the acceleration zone is expected to begin near the $70 \%$ peak magnetic field location. These results agree nicely with the value of $80 \%$ given be Gavryushin et $a l .{ }^{18}$ and give an additional level of texture to the problem. During the design process the magnetic field lines that correspond to the 70 and $90 \%$ magnetic field points should be identified and designed accordingly. These results are more general than the results of the previous section and can apply to a broad range of thrusters. Defining the approximate start of the acceleration zone is valuable information in the early design stages.
Table III. Stepwise Regression Statistics for B-field Fraction at the Acceleration Zone Start

\begin{tabular}{cccc}
\hline \hline & $\begin{array}{c}\text { Primary } \\
\text { Variable } \\
\text { (Peak B) }\end{array}$ & $\begin{array}{c}\text { One-Factor } \\
\text { Regression }\end{array}$ & $\begin{array}{c}\text { w/ Two- } \\
\text { Factor } \\
\text { Interactions }\end{array}$ \\
\hline RSME & 0.03687 & 0.03087 & 0.03008 \\
$\mathrm{R}^{2}$ & 0.698 & 0.8059 & 0.8158 \\
Adjusted R & 0.6477 & 0.7530 & 0.7655 \\
P-value & $1.99 \times 10^{-4}$ & $1.2124 \times 10^{-4}$ & $9.1093 \times 10^{-5}$ \\
\hline \hline
\end{tabular}

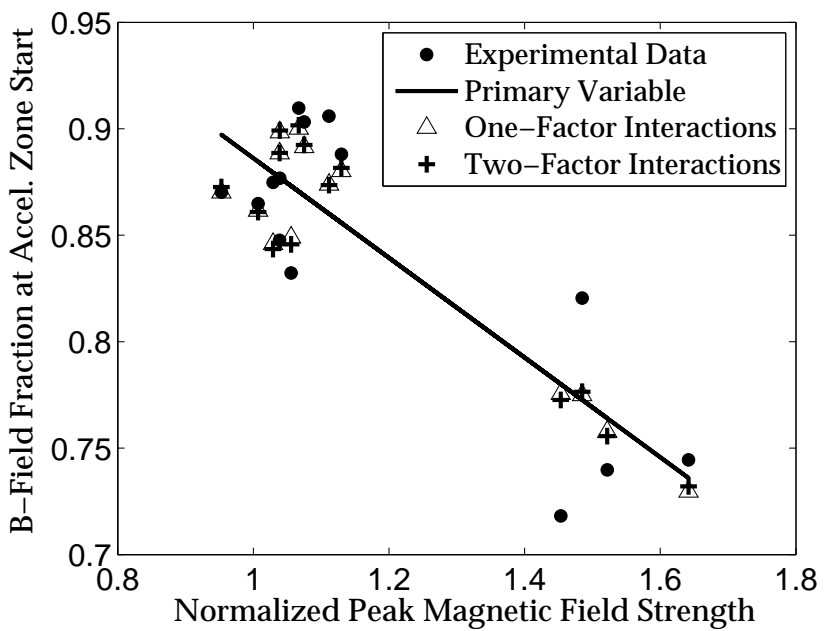

Figure 4. Magnetic Field Fraction at Acceleration Zone Start Regression Results 


\section{Conclusions and Suggestions for Future Work}

This paper offers a novel approach to study the location of the acceleration zone in Hall thrusters. A stepwise regression is used to identify statistically significant trends in these data. These trends were then related back to Hall thruster physics to test the validity of the regression results. The coefficients calculated in the regression equations should not be considered exact values; however the large scale trends are expected to be reliable.

This study finds that the location of the acceleration zone is driven by the cross-field electron mobility and can be controlled by thruster operation parameters. The location of the acceleration zone is found to be mostly dictated by the strength of the magnetic field and the discharge current. The acceleration zone is shown to move upstream with increasing magnetic field strength. There is also a two-factor interaction between discharge current and peak magnetic field strength. As the discharge current term increases, the acceleration zone moves downstream.

With the results of this presented analysis several important design considerations can be suggested. Given approximate operation parameters, the start of the acceleration zone can be estimated. The acceleration zone will start in the region where the centerline magnetic field strength is equal to $70-90 \%$ of the peak centerline magnetic field strength. Since the equipotential lines roughly follow the magnetic field streamlines, the shape of the upstream side of the acceleration zone can also be roughly determined. With this knowledge, it should be possible to predict the relative beam divergence performance of the thruster and the discharge channel erosion location during the thruster design stage.

In the future, a similar analysis could be conducted with carefully designed operation points and greater controls. The thruster should not be subject to ITAR restrictions. This study should cover a range of magnetic field settings, anode flow rates, and should focus on only xenon propellant. Discharge voltage should also be varied but less comprehensively. Discharge channel dimensions, materials, and thruster type could also be varied. The study would only require one centerline sweep with a floating emissive probe and a characterization of the magnetic field structure in the discharge channel. Although a Faraday probe sweep could be included to explore the effect on beam divergence. With carefully designed data points, more detail into the acceleration zone location could be discovered.

\section{Acknowledgements}

J. A. Linnell thanks Prof. Daniel Frey (MIT, Mechanical Engineering and Engineering Systems) for his valuable discussion. This work was supported in part by NASA Glenn Research Center under grant NCC04GA38G (grant monitor Mr. David Jacobson) and from Association François-Xavier Bagnoud.

\section{References}

${ }^{1}$ J. A. Linnell and A. D. Gallimore, "Efficiency Analysis of a Hall Thruster Operating with Krypton and Xenon," Journal of Propulsion and Power, Vol. 22, No. 6, Nov-Dec 2006, pp. 1402-1418.

${ }^{2}$ J. A. Linnell and A. D. Gallimore, "Internal Plasma Potential Measurements of a Hall Thruster Using Plasma Lens

Focusing," Physics of Plasmas, Vol. 13, No. 103504, September 2006.

${ }^{3}$ J. A. Linnell and A. D. Gallimore, "Internal Plasma Potential Measurements of a Hall Thruster Using Xenon and Krypton Propellant," Physics of Plasmas, Vol. 13, No. 093502, September 2006.

${ }^{4}$ R. R. Hofer, R. S. Jankovsky and A. D. Gallimore, "High-Specific Impulse Hall Thrusters, Part 1: Influence of Current Density and Magnetic Field," Journal of Propulsion and Power, Vol. 22, No. 4, July-August 2006, pp. 721-731.

${ }^{5}$ R. R. Hofer, R. S. Jankovsky and A. D. Gallimore, "High-Specific Impulse Hall Thrusters, Part 2: Efficiency Analysis," Journal of Propulsion and Power, Vol. 22, No. 4, July-August 2006, pp. 732-740.

${ }^{6}$ A. I. Morozov, "Focusing of Cold Quasineutral Beams in Electromagnetic Fields," Soviet Physics - Doklady, Vol. 10, No. 8, February 1966, pp. 775-777.

${ }^{7}$ V. V. Zhurin, H. R. Kaufman and R. S. Robinson, "Physics of Closed Drift Thrusters," Plasma Sources Science and Technology, Vol. 8, No. 1, February 1999, pp. R1-R20.

${ }^{8}$ G. J. M. Hagelaar, J. Bareilles, L. Garrigues and J.-P. Boeuf, "Role of Anomalous Electron Transport in a Stationary Plasma Thruster Simulation," Journal of Applied Physics, Vol. 93, No. 1, 15 October 2002, pp. 67-75.

${ }^{9}$ J. M. Fife, "Hybrid-PIC modeling and electrostatic probe survey of Hall thrusters," Doctoral Dissertation, Dept. of Aeronautics and Astronautics, Massachusetts Institute of Technology, Cambridge, MA, 1998.

${ }^{10}$ E. Ahedo, "Radial Macroscopic Model of a Plasma Flowing Along Annual Dielectric Walls," Physics of Plasmas, Vol. 9, No. 7, July 2002, pp. 3178-3186.

${ }^{11}$ R. R. Hocking, "A Biometrics Invited Paper. The Analysis and Selection of Variables in Linear Regression," Biometrics, Vol. 32, No. 1, March 1976, pp. 1-49.

${ }^{12}$ H. Chipman, M. Hamada and C. F. J. Wu, "A Bayesian Variable-Selection Approach for Analyzing Designed Experiments with Complex Aliasing," Technometrics, Vol. 39, No. 4, November 1997, pp. 372-381.

${ }^{13}$ Y. Raitses, D. Staack, M. Keidar and N. J. Fisch, "Electron-Wall Interaction in Hall Thrusters," Physics of Plasmas, Vol. 12, No. 057104, 2005. 
${ }^{14}$ N. Yamamoto, T. Nakagawa, K. Komurasaki and Y. Arakawa, "Effect of Discharge Oscillations on Hall Thruster Performance," 23rd the International Symposium on Space Technology and Science, ISTS 2002-b-17, Shimane, Japan, 2002.

${ }^{15}$ E. Ahedo and D. Escobar, "Influence of Design and Operation Parameters on Hall Thruster Performances," Journal of Applied Physics, Vol. 96, No. 2, July 2004, pp. 983-992.

${ }^{16}$ J. M. Haas and A. D. Gallimore, "Internal Plasma Potential Profiles in a Laboratory-Model Hall Thruster," Physics of Plasmas, Vol. 8, No. 2, February 2001, pp. 652-660.

${ }^{17}$ B. M. Reid and A. D. Gallimore, "Plasma Potential Measurements in the Discharge Channel a 6-kW Hall Thruster," AIAA Joint Propulsion Conference, AIAA-2008-5185, Hartford, CT, 2008.

${ }^{18}$ V. M. Gavryushin, V. Kim, V. I. Kozlov and N. A. Maslennikov, "Physical and Technical Bases of the Modern SPT Development," 24th International Electric Propulsion Conference, IEPC-1995-038, Moscow, Russia, 1995. 